\title{
Efficient Dynamic Channel Allocation Techniques with Handover Queuing for Mobile Satellite Networks
}

Enrico Del Re, Senior Member, IEEE, Romano Fantacci, Senior Member, IEEE, and Giovanni Giambene

\begin{abstract}
Efficient dynamic channel allocation techniques with handover queuing suitable for applications in mobile satellite cellular networks, are discussed. The channel assignment on demand is performed on the basis of the evaluation of a suitable cost function. Geostationary and low earth orbit (LEO) satellites have been considered. In order to highlight the better performance of the dynamic techniques proposed, a performance comparison with a classical fixed channel allocation (FCA) has been carried out, as regards the probability that a newly arriving call is not completely served. It has also been shown that a higher traffic density, with respect to GEO systems, is manageable by means of LEO satellites.
\end{abstract}

\section{INTRODUCTION}

$\mathbf{T}$ HERE is a communication revolution in progress today: the demand for a broad range of telecommunication services (e.g., voice, data, images transmission) is growing and wireless access solutions are attracting because they free the users from tether constraints. Some projects and proposals have been developed to satisfy these pressing needs and to achieve a worldwide network capable of furnishing a wireless access to subscribers wherever they are, using a universal personal address. The challenge here is to implement efficient universal (personal communication services (U-PCS).

In this scenario, tax mobile satellite systems (MSS's) will play a significant role. The development expected for MSS's is based on their capability in managing global coverage systems with a variety of users on the land, in the air, or on the sea. In addition, MSS represents a convenient choice to offer communication services to scarcely populated lands or broad areas where the implementation of a terrestrial mobile network would be unrealizable (for example, oceans) or too expensive. Moreover, many additional advantages seem to be possible by internetworking MSS's and terrestrial networks.

Different solutions to achieve a global coverage MSS are now under investigation. Promising alternative approaches seem to be those resorting to the use of geostationary satellites or low earth orbit (LEO) satellites [1].

Geostationary satellites are fixed with respect to a terrestrial observer and they are on an equatorial circular orbit at about $36000 \mathrm{~km}$ of altitude. Theoretically, only three GEO satellites are required to serve all the Earth.

\footnotetext{
Manuscript received January 15, 1994; revised September 15, 1994. This work was supported in part by the Italian Space Agency and MURST.

The authors are with the Dipartimento di Ingegneria Elettronica, Università di Firenze, 350139 Firenze, Italy.

IEEE Log Number 9407499.
}

On the contrary, LEO satellites are placed on circular or elliptical orbits, at an altitude ranging from 500 to $2000 \mathrm{~km}$. Many LEO satellites are needed to guarantee the coverage of the whole Earth all the day long. Despite this drawback, LEO satellites offer the following advantages over GEO satellites:

1) By using satellites at relatively low altitudes it is less difficult to satisfy the budget link, even with low-power handheld terminals particularly limited in size as well as to reduce propagation delays and cells size.

2) It is possible to cover polar regions with acceptable elevation angles. This is a feature of considerable importance for a global communication system. Note that a mobile subscriber (MS) must see a satellite with an elevation angle greater than $15^{\circ}$ in order to obtain a reliable link.

3) LEO satellites are smaller than GEO satellites: their inorbit mass ranges from 50 to $700 \mathrm{~kg}$. For LEO satellites, a multiple launches arrangement is also possible, thus rendering this solution economically feasible.

The main problem which arises in using a LEO mobile satellite system (LEO-MSS) is due to the satellites motion with respect to a fixed point on the Earth (their orbital speed increases when the altitude decreases).

It is shown in [2] that a convenient representation of the LEO scenario is that of considering any MS moving relatively to a LEO satellite with the speed equal to the orbital satellite velocity $V_{\text {orb }}$. This results from the high value of $V_{\text {orb }}(\approx$ $26000 \mathrm{~km} / \mathrm{h}$ ) with respect to the other motion component speeds (due to the Earth rotation around its axis and to user motion relative to the Earth).

This paper mainly focuses on LEO-MSS's. In particular, the IRIDIUM system proposed by Motorola [3] is considered. The IRIDIUM system uses 66 satellites over six polar circular orbits at about $780 \mathrm{~km}$ of altitude: $V_{\text {orb }}=26600 \mathrm{~km} / \mathrm{h}$.

The coverage area has been divided into cells with each cell illuminated by an antenna spot-beam of a satellite. In this paper, it has been assumed that all the cells are hexagonal regular as shown in Fig. 1. In this figure we have denoted by $R^{\prime}$ the radius of the circle circumscribed on the cell and by $R$ the radius of the circle inscribed in the hexagonal cell. The cell side has been assumed equal to $R^{\prime}$.

In practical applications, parameter $R$ may range from a minimum value $R_{\min }$ (when the satellite is at the zenith) to a maximum value $R_{\max }$ (corresponding to the minimum elevation angle for which the MS can see the satellite), depending on the satellite position with respect to the MS. With the 


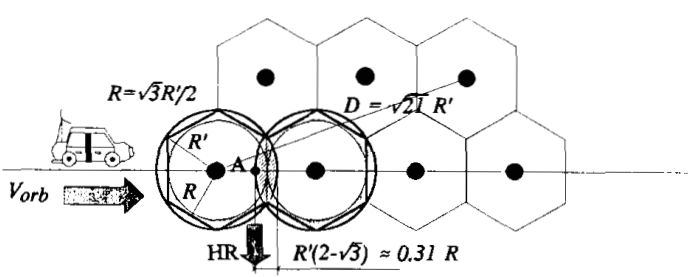

30 = supplementary coverage area for a cell, with radius $\mathbf{R}^{\prime}$

= main coverage area for a cell, with radius $R$

= maximum overlap area between adjacent cells

Mobility assumptions:

- MSs cross the cells along the maximum diameter,

An MS (with a call in progress) leaving cell $\mathrm{x}$, sends a Handover Reguest

(HR), when it enters the overlap area in A.

Fig. 1. Cells and their supplementary coverage areas.

aim of simplifying our analysis, we have assumed $R$ equal to $0.5\left(R_{\max }+R_{\min }\right)$. In particular, under this assumption, we have considered $R$ equal to $212.5 \mathrm{~km}$ in the IRIDIUM case.

Channels are assigned to cells in order to maintain the cochannel interference at an acceptable level. This means that the same sets of channels can be used in different cells with centres distance $D$ (reuse distance) [4].

In a hexagonal regular cellular layout, $D$ can assume only fixed values. The derivation on $\mathrm{D}$ depends on several factors, including the particular multiple access techniques, the modulation scheme used, the mobile environment and the acceptable voice quality [4]. In this paper, we assume $D=\sqrt{21} R^{\prime}$, where $R^{\prime}$ is the cell's side.

A dynamic channel allocation (DCA) is considered as an alternative to the classical fixed channel allocation (FCA) in order to achieve a better channel utilization.

An important problem arising in MSS's is that an MS, with a call in progress, may cross the boundary with an adjacent cell. In this case, a new channel must be assigned to the call in the new cell (destination cell), to avoid a forced call termination. This procedure is called (interbeams) handover [3].

Many handover policies have been recently proposed. Some of them privilege the handover service at the expense of new arrivals. Prioritization schemes are motivated by the fact that, from the MS point of view, a call dropping due to an unsuccessful handover is less desirable than the blocking of a new call attempt [5].

The aim of this paper is to study the joined effect of a dynamic channel allocation technique and of a handover prioritization strategy in order to reduce the probability that a call in progress be dropped $\left(P_{\text {drop }}\right)$.

Some possible prioritization techniques can be based on:

1) guard channels used exclusively to serve handover attempts [5], [7];

2) queuing of handover $(\mathrm{QH})$ requests [6], [7];

3) channel rearrangement techniques (only in the case of dynamic allocation) [2], [8].

In this paper, a handover prioritization strategy based on queuing of handovers is considered. In such a scheme any handover request, arriving at a cell when there are no available channels, can be queued for a fixed maximum time $t_{w}$ max. Elapsed $t_{w} \max$, if no channel becomes available in the destination cell, and the call is still in progress, the handover procedure fails and the associate call is forced into termination.

The performance of this handover queuing scheme has been derived by simulations in the case of a classical FCA technique (FCA-QH) and in DCA techniques (DCA1-QH and DCA2$\mathrm{QH}$ ) in terms of $P_{n s}$, i.e., the probability that a newly arriving call will not be completely served because of initial blocking or unsuccessful handover. An analytical approach has also been developed in the FCA-QH case.

In particular, under the assumption that 1) new call arrivals in the cells are Poisson independent, with a mean rate $\lambda$ per cell, and 2) that the call duration time $t_{d}$ is exponentially distributed with an expected value equal to $T_{m}$, it will be shown in this paper that 1) queuing of handover requests significantly allows to reduce $\left.P_{n s}, 2\right) P_{n s}$ decreases when mobility decreases.

This paper is organized as follows: Section II describes the mobility model assumed for the LEO systems and the protocol employed in managing handover attempts. In Section III, the problem of an efficient channel allocation among users is considered. An analytical approach for evaluating the FCAQH performance is presented in Section IV. Finally, Section V deals with simulation results, concerning the performance of dynamic techniques and FCA both for GEO and LEO systems, either with or without queuing of handover requests.

\section{MOBILITY MODEL AND QUEUING OF HANDOVER REQUESTS}

System performance is generally dependent on the handovers management technique used. According to the link quality measurement approach [5], we have assumed in our study that a handover must be performed to avoid the forced termination of the associated call in the time interval during which the ratio of the power level received from the spotbeam irradiating the current cell of the MS and the spotbeam irradiating its destination cell is within two appropriate thresholds.

The higher threshold is set at the point where the power received from the spot-beam irradiating the destination cell has started to increase at the expense of a reduction of the power received from the spot-beam illuminating the current cell of the MS. Likewise, the lower threshold is the point at which the power received by the MS from the current spot-beam is at the minimum acceptable level.

The area on which an MS can receive a signal with an acceptable power level from two adjacent spot-beams is named overlap area.

The extension of the overlap area is a parameter that directly affects the system performance. Obviously, a wider overlap area leads to a lower handover failure probability, under specified traffic load conditions. The extension of this region is dependent on the direction of MS (relative to satellite) motion, the satellite antenna characteristics, and the propagation conditions. Then, taking into account that for the 


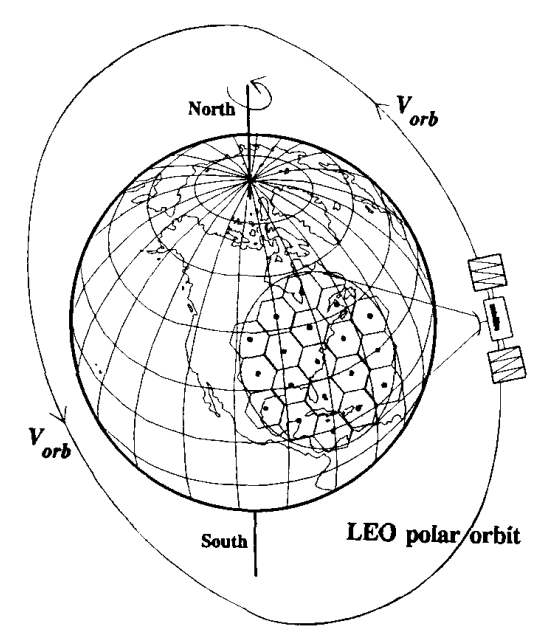

Fig. 2. Cellular layout orientation with respect to satellite motion.

assumed satellite cellular network configuration (Fig. 1), the maximum extension of the overlap area is equal to $R^{\prime}(2-$ $\sqrt{3}) \approx 0.31 R$, we have assumed a maximum extension of the overlap area equal to $R / 5$ as a conservative choice [6].

The impact of handovers on system performance depends on the orbit type chosen in MSS (e.g., GEO or LEO).

In the GEO case, the cells illuminated by a satellite are fixed (stationary) with respect to a point on the Earth; then, handovers are produced only by the MS motion with respect to the Earth. Moreover, the average call duration is negligible in comparison with the cell crossing times (with MS speed). This means that the handover occurrence is very low, and therefore we can consider, with good approximation, that a user (called "fixed" user) does not change cell.

On the contrary, in LEO systems, handovers occur frequently, making it necessary to resort to efficient handover policies. In performing our analysis in the case of LEO-MSS, we have called "source cell" the cell where the MS call begins and any cell, reached by the associated MS during the call lifetime, was named "transit cell."

We consider that an antenna from a satellite with honeycomb arrangement of spot-beams produces a cellular pattern like that shown in Fig. 2, i.e., with the maximum diameter in the north-south direction.

Moreover, the following assumptions have been made (see Fig. 1):

1) MS's cross cells at a constant velocity (relative to the satellite) equalling satellite orbital speed $V_{\text {orb }}$ [2].

2) Every MS crosses the cells along their maximum diameter. ${ }^{1}$

3) When a handover occurs, the destination cell is the neighboring cell in the direction of satellite motion.

4) From call outset in a cell, MS travels a distance that can be assumed as a) uniformly distributed between 0

'We have neglected the case of an MS crossing the cells along the seam of the cellular layout. In this case the MS moves essentially along overlap areas of adjacent cells. However, the actual number of handovers is usually less than the number of crossed cells, therefore having a slight impact on the results obtained under our approximation. and $2 R$, if the cell is the "source cell" for the call, 2) deterministically equal to $2 R$, if the call is in a "transit cell."

The user mobility is characterized in this paper by the parameter $\alpha$, defined as

$$
\alpha \triangleq \frac{2 R}{V_{\text {orb }} T_{m}^{\top}} .
$$

Parameters $R$ and $V_{\text {orb }}$ depend on the satellite constellation altitude; moreover, $R$ is also depending on the half power beam width (HPBW) of the spot-beams from the satellite antenna. Considering a fixed value for $T_{m}$, we have assumed that the MS mobility increases if $V_{\text {orb }}$ increases and/or $R$ decreases (i.e. $\alpha$ decreases) [2].

This paper deals with a particular handover prioritization scheme based on the possibility of queuing the handover requests, when the associated MS is within the overlap area (= handover area).

We have assumed that the service discipline is of the FIFO type and a handover may be cleared from the queue, whenever the associate MS goes out from the handover area.

An MS having a call in progress in a certain cell $x$ sends a handover request as soon as it enters the handover area (Fig. 1). According to the assumed mobility model. we can derive the maximum value of the waiting time for any handover request $t_{w} \max$

$$
t_{w \max }=\frac{\alpha T_{m}}{10}
$$

We stress that $t_{w} \max$ is the maximum available time to satisfy a handover request, but this does not represent the time really spent by a call in the overlap area, since the call may end before leaving this region.

Let $t_{m c 1}$ be the interval elapsed from the arrival instant of a new call for an MS in a cell (source cell) to the instant in which MS enters the handover area. On the basis of mobility assumptions, $t_{m c 1}$ is a random variable uniformly distributed between 0 and $2 R / V_{\text {orb. }}$.

The random call duration time $t_{d}$ is exponentially distributed, with mean $T_{m}$. An MS with a call in progress in its source cell gives rise to a handover request whenever $t_{d}>t_{m c 1}$. The probability of this event $P_{h 1}$ is

$$
P_{h l}(\alpha)=\operatorname{Pr}\left\{t_{d}>t_{m c 1}\right\}=\frac{1-e^{-\alpha}}{\alpha} .
$$

An MS having a call in progress, which has already been handed off with success, must travel a deterministic distance equal to $2 R$ with velocity $V_{\text {orb }}$ in a transit cell, before sending a new handover request. Then, $t_{m c 2}\left(=2 R / V_{\text {orb }}\right)$ represents the deterministic interarrival time for the handovers subsequent to the first.

Due to the memoryless property of the exponential distribution, the residual service time of a call after a successful handover request has the same probability density function as $t_{d}$. It follows that the probability that an MS, with a call in progress, sends a new handover request $P_{h 2}$ is given by

$$
P_{h 2}(\alpha)=\operatorname{Pr}\left\{t_{d}>t_{m c 2}\right\}=e^{-\alpha} .
$$




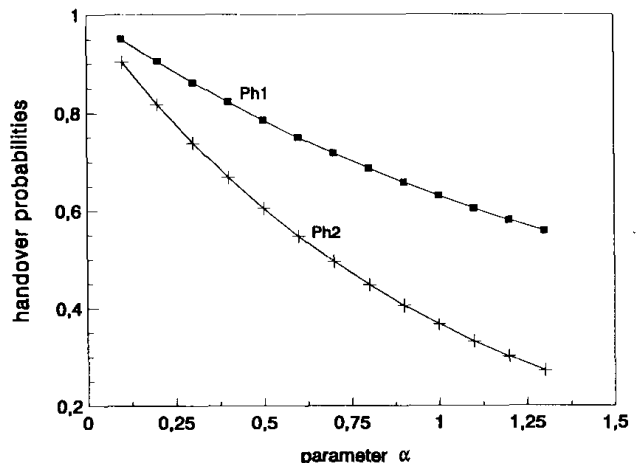

Fig. 3. Handover probabilities as a function of $\alpha$, the mobility parameter.

Parameters $P_{h 1}$ and $P_{h 2}$ are shown in Fig. 3 as a function of $\alpha$. It is evident in this figure that as $\alpha$ approaches $0(\infty), P_{h 1}$ and $P_{h 2}$ approach $1(0)$.

From the above considerations it can be easily noted that the handover requests generation is memoryless. We take into account the following:

1) $P_{b 1}$, blocking probability for new call attempts,

2) $P_{b 2}$, handover failure probability,

3) $Q_{i}$, probability that a call lasts so as to produce at least $i$ handovers $(i=1,2, \cdots)$, defined as

$$
Q_{i}=P_{h 1} P_{h 2}^{i-1} \text {, }
$$

4) $V_{i}$, probability that a call attempt is served and has $i$ successful handovers $(i=1,2, \cdots)$, defined as

$$
V_{i}=\left(1-P_{b 2}\right)^{i-1}\left(1-P_{b 1}\right),
$$

5) $B_{i}$, probability that a served call is dropped to the $i$ th handover $(i=1,2, \cdots)$, defined as

$$
B_{i}=\left(1-P_{b 2}\right)^{i-1} P_{b 2} \text {. }
$$

Therefore, the mean value of the number of times that a newly arriving call is successfully handed off during its lifetime $n_{h}$ can be derived as

$$
n_{h}=\sum_{i=1}^{\infty}\left\{i Q_{i} V_{i}\right\}=\frac{\left(1-P_{b 1}\right) P_{h 1}}{1-\left(1-P_{b 2}\right) P_{h 2}} .
$$

Fig. 4 shows $n_{h}$ in the case $P_{b 1}=P_{b 2}=0$, as a function of LEO satellites altitudes, according to the mobility model and assuming a constant call arrival rate per cell and an HPBW of the antenna spot-beams on satellites, whatever their altitude. Parameter $n_{h}$ increases as the LEO altitude decreases. Then, handover prioritization policies are more important when satellites altitude decreases.

To clearly point out the advantages offered by handover queuing, the system performance is evaluated in terms of probability $P_{n s}$, i.e., the probability that a call is cleared because it is blocked at its initial attempt or because it is dropped because of unsuccessful handover [7]. Probability $P_{n s}$ can be defined as

$$
P_{n s}=P_{b 1}+\left(1-P_{b 1}\right) P_{\text {drop }}
$$

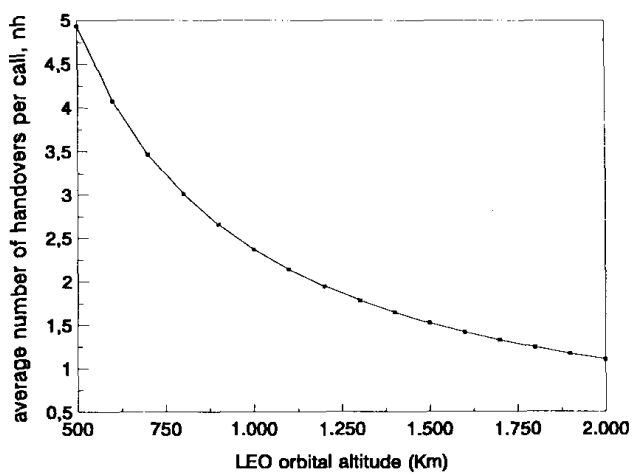

Fig. 4. Average number of handovers per call as a function of the LEO orbital altitude

where $P_{\text {drop }}$, the probability that a call in progress be dropped, due to handover failure, can be derived, according to our mobility model, as follows

$$
P_{\text {drop }}=\sum_{i=1}^{\infty}\left\{Q_{i} B_{i}\right\}=\frac{P_{h 1} P_{b 2}}{1-P_{h 2}\left(1-P_{b 2}\right)} .
$$

The channel holding time in a cell can be derived as

$$
t_{H i}=\min \left[t_{d}, t_{m c i}\right]
$$

where $i=1$, for a call in its source cell, $i=2$, for a call in its transit cells. From (11) the expected value of $t_{H i}, E\left[t_{H i}\right]$ results in

$$
E\left[t_{H i}\right]=T_{m}\left(1-P_{h i}\right) .
$$

In our study the handover arrivals process has been modeled as a Poisson process, independent of the new arrivals process and with average arrival rate $\lambda_{h}$, related to $\lambda$ by

$$
\lambda\left(1-P_{b 1}\right) P_{h 1}+\lambda_{h}\left(1-P_{b 2}\right) P_{h 2}=\lambda_{h} .
$$

Equation (13) has been derived by considering a statistical equilibrium between MS's leaving a cell and MS's entering the cell. Finally, we note that from (13) and (8) we have

$$
\frac{\lambda_{h}}{\lambda}=n_{h}
$$

\section{Allocation TeChniques}

In this section, the classical FCA technique and two alternative solutions for achieving a dynamic channel assignment in an MSS are discussed. The case of an FCA technique is first considered.

\section{A. Fixed Channel Allocation (FCA)}

In an FCA technique [2], [4], [8] a set of channels is permanently assigned to each cell. The same set of channels is reused in cells at a distance $D$ away. The basic FCA technique implies that a call attempt at a cell can only be served by an available channel belonging to the set of channels assigned to that cell. If no channel is available the call is blocked and lost. 


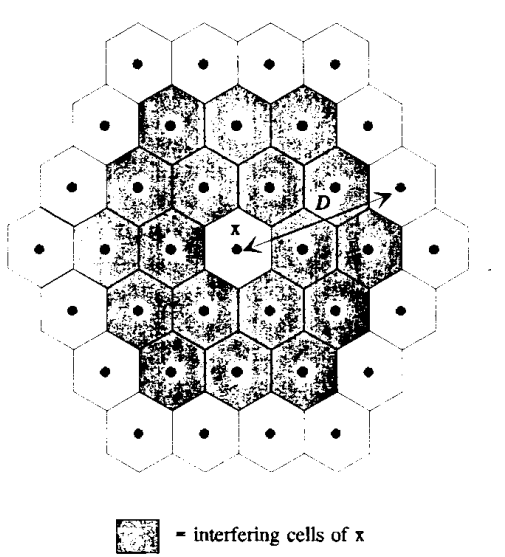

Fig. 5. Cell $x$ and its interfering cells, for a hexagonal cellular layout, with $D=\sqrt{21} R^{\prime}\left(R^{\prime}=\right.$ cells side $)$.

The number of channels permanently allocated to each cell $S$ can be derived as [4]

$$
S=\frac{M}{K}
$$

where $M=$ number of system resources; $K=D^{2} /\left(3 R^{\prime 2}\right)$, the reuse factor.

\section{B. Dynamic Channel Allocation}

A dynamic channel assignment permits an efficient spectrum utilization, because each channel may be used in any cell of the network, provided that the constraint on $D$ is respected. In order to serve a new call attempt, a certain cell $x$ can only use the channels just not used either in $x$ or in $I(x)$, where $I(x)$ denotes the cells distant less than $D$ from $x$ (i.e., interfering cells of $x$ ). Fig. 5 shows the cells $\in I(x)$, for $D=\sqrt{21} R^{\prime}$.

Let us denote with $\Lambda(x)$ the set of available channels for cell $x$ at the call arrival instant in $x$.

When an arrival must be served in cell $x$, if it results $\Lambda(x) \neq \varnothing$, the best channel to be allocated is selected on the basis of the evaluation of a suitable cost function $C_{x}(i)$, $i \in \Lambda(x)$, according to the following minimum cost function criterium:

channel $i^{*}$ is allocated to cell $x$, if

$$
C_{x}\left(i^{*}\right)=\min _{i \in \Lambda(x)}\left\{C_{x}(i)\right\} .
$$

If more channels verify (16), a random choice is performed.

In the following, two possible alternatives are considered to evaluate the cost function of any channel $i \in \Lambda(x), C_{x}(i)$, called, respectively, "DCA1" and "DCA2."

1) "DCAl" Technique: In the case of the DCA1 technique, channel $i$, which becomes locked in the minimum number of cells $\in I(x)$, is allocated to cell $x$. Therefore, the allocation cost function can be defined as [2], [8], [9]

$$
C_{x}(i) \triangleq \sum_{k \in I(x)}\left\{u_{k}(i)\right\}, \quad \forall i \in \Lambda(x)
$$

where

$$
u_{k}(i)= \begin{cases}1, & \text { if } i \in \Lambda(k) \\ 0, & \text { otherwise }\end{cases}
$$

The DCAl defined by (17) and (18) performs only a local optimization for the channel assignment. This leads to an inefficient channel distribution in the network, in particular under heavy traffic conditions, i.e., the minimum channel reuse distance is much greater than $D$. This gives rise to a substantial increase in call blocking, even greater than with FCA, as will be shown later (see Figs. 8 and 12). To overcome this problem, the DCA2 technique described below has been proposed.

2) "DCA2" Technique: In the DCA2 case, we start with a fixed channel allocation, according to the reuse distance $D$. Then we denote by $F_{D}(x)$ the set of channels allocated according to FCA to cell $x$. We stress that a fixed allocation assures a distribution of channels among the cells of the network, with the minimum possible reuse distance $D$. With the proposed DCA2 technique, in performing a dynamic allocation of a channel in cell $x$, whenever possible, we select channels belonging to $F_{D}(x)$.

Therefore, the allocation cost contribution for channel $i \in$ $\Lambda(x)$, due to the interfering cell $k \in I(x), C_{x}(k, i)$, can be expressed as

$$
C_{x}(k, i)=u_{k}(i)+2\left(1-q_{k}(i)\right), \quad \forall k \in I(x)
$$

where $u_{k}(i)$ is defined in (18) and $q_{k}(i)$ is given by

$$
q_{k}(i)= \begin{cases}0, & \text { if } i \in F_{D}(k) \\ 1, & \text { otherwise. }\end{cases}
$$

In defining the cost function $C_{x}(k, i)$, relative to the status of channel $i$ in cell $k \in I(x)$, the first term takes into account the availability of channel $i$ in cell $k$, while the second term represents channel $i$ belonging to the optimal set for cell $k$ (i.e., $F_{D}(k)$ ). In addition to this, we have chosen to weight this second aspect with a factor 2 , in order to follow as much as possible the FCA channel distribution. Note that $C_{x}(k, i)$ can assume only four different values

$$
C_{x}(k, i)= \begin{cases}0, & \text { if } i \notin \Lambda(k) \text { and } i \notin F_{D}(k) \\ 1, & \text { if } i \in \Lambda(k) \text { and } i \notin F_{D}(k) \\ 2, & \text { if } i \notin \Lambda(k) \text { and } i \in F_{D}(k) \\ 3, & \text { if } i \in \Lambda(k) \text { and } i \in F_{D}(k) .\end{cases}
$$

Therefore, the overall cost function can be obtained as

$$
C_{x}(i) \triangleq q_{x}(i)+\sum_{k \in I(x)}\left\{C_{x}(k, i)\right\}, \quad \forall i \in \Lambda(x)
$$

where the term $q_{x}(i)$ is introduced in (22) to take into account that it is preferable to allocate in $x$ a channel $i$ belonging to the nominal (FCA) channels set of $x$ (i.e., $i \in F_{D}(x)$ ). In particular, $q_{x}(i)$ has been introduced only to discriminate among different situations with equal cost for the interfering cells, in order to allocate in $x$, if possible, a channel $\in F_{D}(x)$.

For both dynamic allocation techniques (i.e., DCAl and DCA2), in order to improve their performance, whenever a call termination occurs in a cell $x$ (due either to the end of the call or to handover), the channel assignments in $x$ must be rearranged to deallocate in $x$ the channel at maximum cost.

Without this rearrangement technique, the performance of DCA1 and DCA2 worsen considerably. For example, if we consider in the LEO-MSS case the DCA2 technique with 


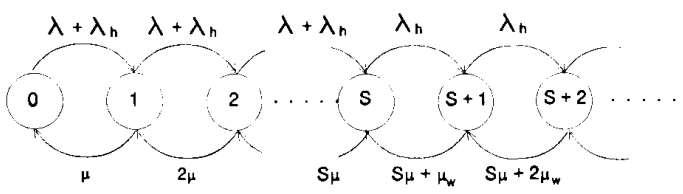

Fig. 6. Markov model for FCA-QH.

Queuing of handovers requests (DCA2-QH) and a uniform traffic of 7.5 erl per cell (see simulation assumptions in Section V), with the proposed rearrangement technique we have $P_{b 1} \approx 3 \%$ and $P_{b 2} \approx 0.2 \%$ (see Fig. 10), but without rearrangements at the call termination in a cell we obtain $P_{b 1} \approx 12 \%$ and $P_{b 2} \approx 0.5 \%$.

\section{ANALYSIS OF FCA-QH}

In this section an analytical approach for evaluating the FCA-QH performance is presented. In performing our analysis we have assumed that

1) $S$ channels/cell are assigned according to (15);

2) new arrivals and handover attempts are two independent Poisson processes, with mean rates $\lambda$ and $\lambda_{h}$, with $\lambda_{h}$ related to $\lambda$ by (14);

3 ) with or without queuing of handovers, the channel holding time in a cell (both for new arrivals and handovers) is approximated as exponentially distributed with mean, $1 / \mu$, expressed as

$$
\frac{1}{\mu}=\frac{\lambda}{\lambda+\lambda_{h}} E\left[t_{H 1}\right]+\frac{\lambda_{h}}{\lambda+\lambda_{h}} E\left[t_{H 2}\right]
$$

where $E\left[t_{H 1}\right]$ and $E\left[t_{H 2}\right]$ are derived from (12);

4) the maximum waiting time is approximated as a random variable exponentially distributed, with expected value $t_{w} \max =\alpha T_{m} / 10 ;^{2}$

5) infinite queue length;

6) the event that a call may be ended when the associated MS is within the handover area has been neglected. The goodness of this assumption will be verified later by comparing simulation results with analytical predictions.

From the above assumptions it follows that in the case of the FCA-QH technique each cell can be modeled as an $\mathrm{M} / \mathrm{M} / \mathrm{S}$ queuing system with nonhomogeneous arrival rates [5] ( $M$ : Poisson arrival process/ $M$ : service time exponentially distributed $/ S$ : number of channels assigned per cell).

We stress that in the simulations $t_{w}$ max is deterministic (according to a more realistic mobility model), while in this study $t_{w}$ max is exponentially distributed. Therefore, the model in Fig. 6 is memoryless, even if the more realistic simulated system is with memory. The approximation made in our analysis has been validated by the results shown at the end of this section.

The status of the queuing system under consideration has been defined as the sum of the number of calls in service and the number of queued handovers. Whenever the system is in a status $n$ less than $S$, the gross arrival rate is $\lambda+\lambda_{h}$; while,

${ }^{2}$ The approximation of the deterministic value $t_{u} \max$, by means of an exponential distribution with expected value $t_{w}$ max, allows a good fit, close to the optimum [7]. if the status is greater than $S$, i.e., all channels are busy, the gross arrival rate is $\lambda_{h}$ (Fig. 6).

When the system is in the state $S+i$, for $i=1,2, \cdots$, to take into account that a handover request may be cleared before attaining service, we have introduced the additional death rate $i \mu_{w}$ (Fig. 6).

The probability of state $n, P_{n}$, can be derived as

$$
P_{n}= \begin{cases}\frac{\left(\lambda+\lambda_{h}\right)^{n}}{n ! \mu^{n}} P_{0}, & 1 \leq n \leq S-1 \\ \frac{\left(\lambda+\lambda_{h}\right)^{S} \lambda_{h}^{n-S}}{S ! \mu^{S} \prod_{j=1}^{n-S}\left(S \mu+j \mu_{w}\right)} P_{0}, & n \geq S\end{cases}
$$

where $\mu_{w}=1 / t_{w} \max$ and the idle system probability $P_{0}$ is given by

$$
\begin{aligned}
P_{0}=\left\{\sum_{n=0}^{S-1}\left[\frac{\left(\lambda+\lambda_{h}\right)^{n}}{n ! \mu^{n}}\right]\right. \\
\left.\quad+\sum_{n=S}^{\infty}\left[\frac{\left(\lambda+\lambda_{h}\right)^{S} \lambda_{h}^{n-S}}{S ! \mu^{S} \prod_{j=1}^{n-S}\left(S \mu+j \mu_{w}\right)}\right]\right\}^{-1}
\end{aligned}
$$

New arrivals are blocked when all the available channels (servers) are in use in the cell, i.e., when the queuing system is in the state $n \geq S$. Therefore, $P_{b 1}$ results in

$$
P_{b 1}=\sum_{n=S}^{\infty} P_{n}
$$

We assume that a handover request occurs when the system (cell) is in the state $n$. The service discipline for queued handovers is FIFO.

Then 1) if $n<S$, the handover is immediately served; 2) on the contrary, if $n \geq S$, the handover request is queued and it is blocked only if the waiting time exceeds a random time derived from an exponential distribution with mean $1 / \mu_{w}$. Therefore, for $n \geq S$ we may define $P_{b 2 \mid n}$ as the handover failure probability conditioned on $n$, that is, the queue state when the handover attempt arrives.

According to [7], for $n \geq S, P_{b 2 \mid n}$ is expressed as

$$
P_{b 2 \mid n}=1-\prod_{j=0}^{n-S}\left[1-\frac{\mu_{w}}{\left(S \mu+\mu_{w}\right) 2^{j}}\right] .
$$

Therefore, $P_{b 2}$ results in

$$
P_{b 2}=\sum_{n=S}^{\infty} P_{b 2 \mid n} P_{n}
$$

A recursive approach is needed to compute $P_{b 1}$ and $P_{b 2}$, because $\lambda_{h}$ is related to $P_{b 1}$ and $P_{b 2}$, by (14). Therefore, we can derive $P_{n s}$ from (9).

Fig. 7 shows $P_{n s}$ in the case FCA-QH, as a function of the traffic intensity due to new arrivals $\left(=\lambda T_{m}\right)$. Simulation results are also reported in that figure to highlight a good agreement with analytical predictions. 


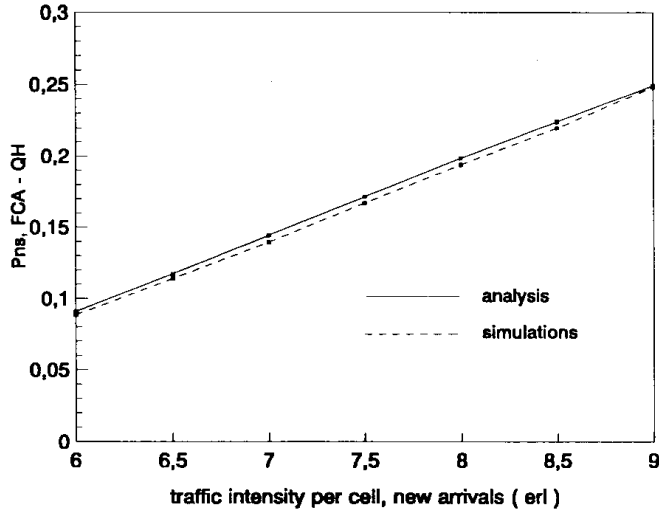

Fig. 7. Analysis and simulation results for FCA-QH, LEO case with $\alpha \approx 0.32$.

TABLE I

System Parameters Assumed in Performing Our Simulations and ANalysis

two tiers of interfering cells (i.e. $D=\sqrt{21} R^{\prime} ; K=7$, for FCA); $M=70$ channels ( 10 channels are assigned per cell with FCA); $T_{m}=3 \mathrm{~min}$, average call duration;

$T_{m}=3 \mathrm{~min}$, average call duration; $425 \mathrm{Km}$ and $V_{\text {orb }}=26,600 \mathrm{Km} / \mathrm{h}$. Then, we obtain $\alpha \approx 0.32$; when queuing handover is allowed, the maximum number of requests waiting service is fixed equal to 10 . This is a good approximation of the infinite queue length, for the traffic range under consideration.

\section{Performance Evaluations and COMPARISONS}

In this section, the performance of the allocation techniques has been derived by simulations. In particular, we have considered an entire parallelogram-shaped cellular network with $N$ cells per side and the results shown in graphics have been gathered only from the central cells, i.e., those cells with a complete interfering cell set in the network. In the following simulations, we use $N=7$ and we refer to system parameters summarized in Table I.

1) LEO-MSS Case: The performance of the FCA and DCA techniques is shown in Figs. 8 and 9 in terms of $P_{b 1}$, and $P_{b 2}$, respectively, for the case without and with queuing of handover requests. Note that by means of FCA, if handovers are not queued, they have the same service priority as that of new arrivals; therefore, $P_{b 1}=P_{b 2}$, and Fig. 8 shows a single curve for FCA.

Queuing of handovers causes a considerable decrease of $P_{b 2}$, at the expense of an increased $P_{b 1}$ : handover prioritization leads to higher blocking values for new arrivals and poorer network traffic carrying out capacity.

To overcome this problem we use the DCA2 technique. Then, Fig. 10 shows the results for DCA1-QH and for DCA2$\mathrm{QH}$ : the new allocation cost significantly reduces $P_{b 1}$, keeping $P_{b 2}$ practically unvaried. Therefore, more traffic is accepted by the network and queuing prevents an increase in handover failures. The DCA2 solution works better than FCA and DCA1 for all the examined traffic loads per cell.

Fig. 11 shows the comparison results in terms of $P_{n s}: P_{n s}$ decreases substantially (at the same traffic load) using the DCA2 allocation cost and queuing of handovers.

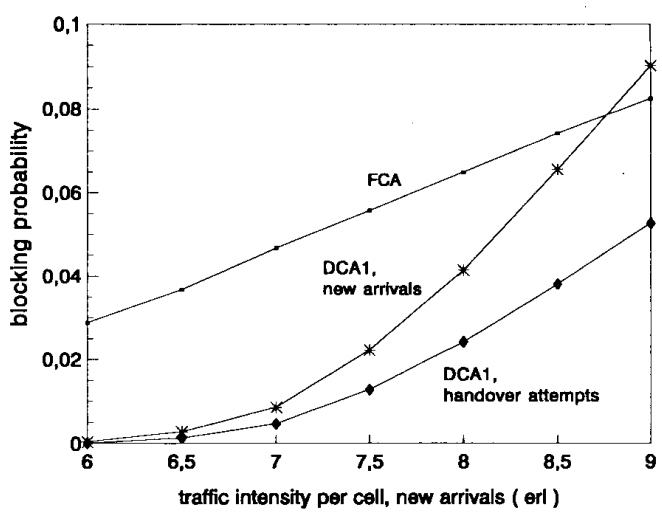

Fig. 8. FCA versus DCAl, LEO case with $\alpha \approx 0.32$.

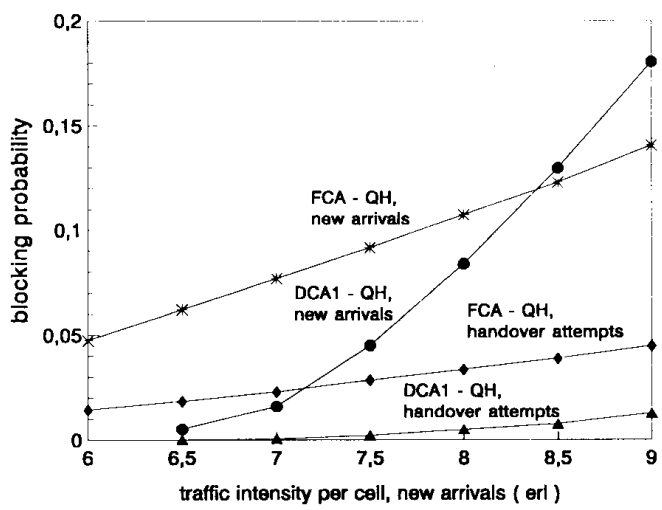

Fig. 9. FCA-QH versus DCA1-QH, LEO case with $\alpha \approx 0.32$.

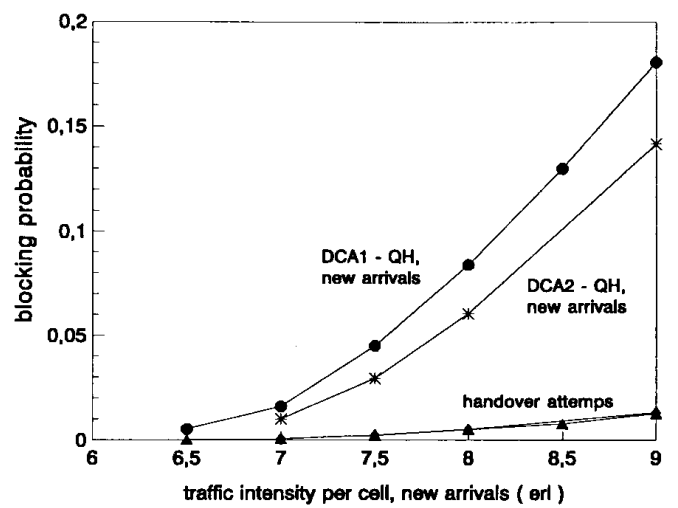

Fig. 10. DCA1-QH versus DCA2-QH, LEO case with $a=0.32$.

2) GEO-MSS Case: We have assumed fixed users $\left(P_{h 1}=\right.$ $P_{b 2}=0$ ) and then $P_{b 2}=P_{\text {drop }}=0, P_{n s}=P_{b 1}$. Fig. 12 shows the behaviors of $P_{n s}$ for FCA, DCA1, DCA2. DCA1 outperforms FCA for medium-low traffic loads, but in the presence of congestion there is a trend inversion. On the contrary, DCA2 exhibits a call blocking probability lower than DCA1 and FCA over all the examined traffic range.

3) GEO versus $L E O$ Solution: Thanks to $P_{n s}$, it is possible to compare LEO and GEO solutions for given system 


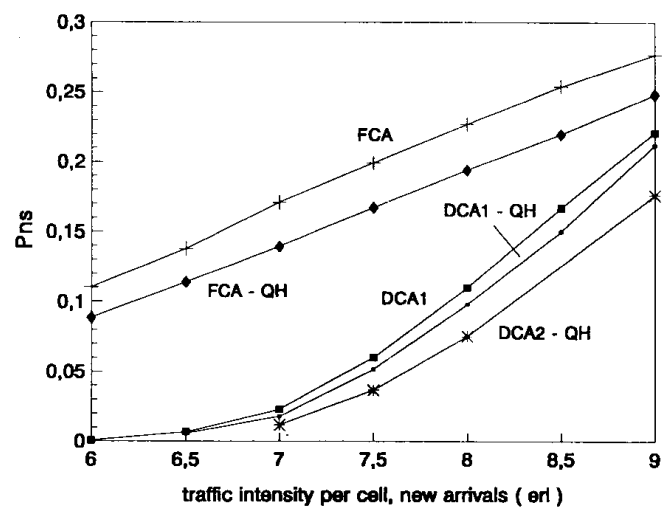

Fig. 11. Comparison among all the allocation techniques presented, in terms of $P_{n s}$, LEO case with $\alpha \approx 0.32$.

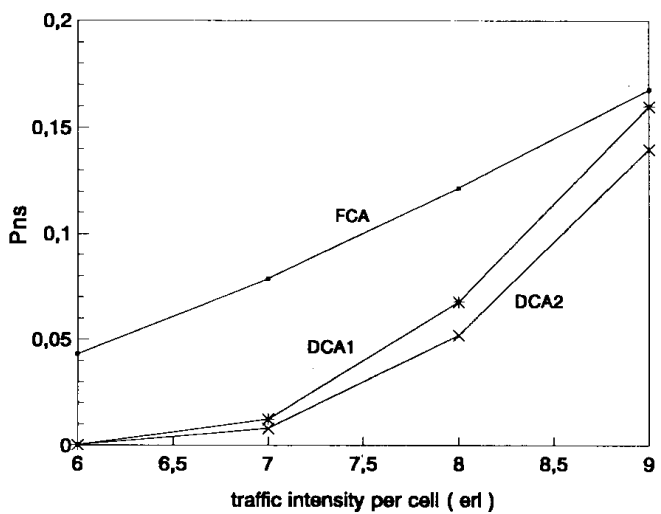

Fig. 12. Comparison among FCA, DCA1, and DCA2 for GEO-MSS

TABLE II

CAPACITY COMPARISON FOR $P_{n s}=3 \%, 20 \mathrm{merl} / \mathrm{user}$, $M=70$ Channels, Two Tiers of Interfering Cells

\begin{tabular}{|c|c|c|c|c|c|}
\hline \multirow{4}{*}{$\begin{array}{l}\text { LEO-MSS } \\
\alpha=0.32\end{array}$} & Allocation & users/cell & \multirow{4}{*}{$\begin{array}{c}\text { GEO-MSS } \\
\qquad(\alpha \rightarrow \infty)\end{array}$} & Allocation & users/cell \\
\hline & $\mathrm{FCA}-\mathrm{QH}$ & 238 & & $\mathrm{FCA}$ & 275 \\
\hline & $\mathrm{DCA} 1-\mathrm{QH}$ & 362 & & DCA I & 365 \\
\hline & $\mathrm{DCA} 2-\mathrm{QH}$ & 368 & & $\mathrm{DCA} 2$ & 375 \\
\hline
\end{tabular}

resources, allocation techniques and reuse distance, in order to evaluate the impact of mobility on system performance. The preceding graphics highlight that we obtain higher values of $P_{n s}$ in LEO systems than in GEO systems. This difference depends on high LEO mobility compared with negligible GEO mobility, and on handover management protocols.

Assuming $P_{n s}=3 \%$ and $20 \mathrm{merl} /$ user [10], the number of potential users per cell is shown in Table II, both for GEOand LEO-MSS. It is a straightforward matter to note that the GEO solution permits achievement of a higher capacity per cell with respect to the LEO solution. However, this statement is overturned if we consider the user's density, when cell sizes are much greater in GEO-MSS $(R \approx 1000 \mathrm{~km})$ than in LEO-MSS $(R \approx 200 \mathrm{~km})$. Another important parameter to be considered is the system implementation cost. Different opinions can be found in the literature, mainly depending on satellite technology, the number of required satellites, and satellite launch costs. From the previous observations, we can consider that LEO-MSS and GEO-MSS share approximately the same implementation cost [11]. Therefore, it results that LEO-MSS represents a better solution than GEO-MSS.

\section{CONClusions}

In this paper, some alternatives to achieving a dynamic channel allocation in MSS's were proposed. The cases of GEO and LEO satellite systems were considered, and the user mobility relative to these MSS's was also discussed. The techniques proposed, namely, DCA1-QH and DCA2$\mathrm{QH}$, make use of a handover prioritization scheme based on queuing of handover requests to obtain a better service quality.

A performance comparison with a classical FCA with handover queuing was also carried out to highlight the better performance of these dynamic channel allocation techniques.

Moreover, the results shown in this paper clearly point out that the LEO solution permits to achieve a higher traffic density than GEO systems and, therefore, it will satisfy the growing wireless access demand with a minimum spectrum resource.

\section{REFERENCES}

[1] J. J. H. Lodge, "Mobile satellite communication systems: Toward global personal communications," IEEE Commun. Mag., vol. 29, pp. 24-30, Jan. 1991.

[2] E. Del Re, R. Fantacci, and G. Giambene, "Performance analysis of a dynamic channel allocation technique for satellite mobile cellular network," Int. J. Satellite Commun., vol. 12, pp. 25-32, Jan./Feb. 1994.

[3] G. Maral, J-J. De Ridder, B. G. Evans, and M. Richharia, "Low Earth orbit satellite systems for communications," Int. J. Sat. Commun., vol. 9, pp. $209-225,1991$.

[4] V. H. MacDonald, "The cellular concept," Bell. Syst. Tech. J., vol. 58, pp. 15-41, Jan. 1979

[5] S. Tekinay and B. Jabbari, "Handover and channel assignment in mobile cellular networks," IEEE Commun. Mag., vol. 29, no. 11, pp. 42-46, Nov. 1991

[6] _ , "A measurement prioritization scheme for handovers in mobile cellular networks," IEEE J. Select. Areas Commun., vol. 10, no. 8, pp. 1343-1350, Oct. 1992

[7] D. Hong and S. S. Rappaport, "Traffic model and performance analysis for cellular mobile radio telephone systems with prioritized and nonprioritized handoff procedures," IEEE Trans. Veh. Technol, vol. VT-35, no. 3, pp. 77-92, Aug. 1986

[8] E. Del Re, R. Fantacci, and G. Giambene, "Performance analysis of a dynamic channel allocation technique for terrestrial and satellite mobile cellular networks," in Proc. GLOBECOM '93 (Houston, TX), Nov. 29-Dec. 2, 1993, pp. 1698-1702.

[9] S. Nanda and D. J. Goodman, "Dynamic resource acquisition: Distributed carrier allocation for TDMA cellular systems," in Proc. GLOBECOM '91 (Phoenix, AZ), Dec. 2-5, 1991, pp. 883-888.

[10] E. Del Re, "Satellite system integrated with the terrestrial cellular network for mobile communications," ESA Tech. Rep., ESA STR-228, Aug. 1989

[11] J. G. Schoenenberger, "Satellite personal communications networks," in Proc. 3rd IEE European Conf. Satellite Commun. (Manchester, UK), Nov. 2-4, 1993, pp. 128-132.

Enrico Del Re (M'78-SM'84) for a photograph and biography, please see the Guest Editorial of this JourNal, p. 178. 


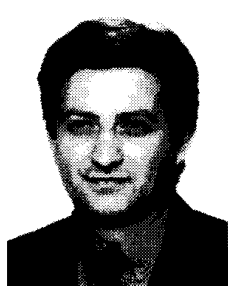

Romano Fantacci (S'84, M'88, SM'91) received the Dr.Ing. degree in electrical engineering from the University of Florence, Florence, Italy, in 1982 and the Ph.D. degree in electrical engineering in 1987.

In 1982, he joined the Department of Electrical Engineering, University of Florence, first as a Research Assistant and presently as an Associate Professor of Telecommunication Networks.

His research interests involve digital communications, queueing systems, multiple access systems, and personal communication networks.

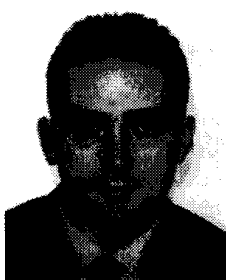

Giovanni Giambene was born in Florence on $\mathrm{Au}$ gust 16,1966 . He received the Dr.Ing. degree in electronics from the University of Florence, Florence, Italy, in 1993. Currently, he is a candidate for the Ph.D. degree in telecommunications and informatics in the Department of Electric Engineering of the same university.

He also acts as the Technical External Secretary of the European Community Project, COST 227 (Integrated Space/Terrestrial Mobile Networks). His research interests are mobile cellular communication networks, personal communication services, channel allocation techniques, and queuing theory. 